\title{
Laplacian gauge and instantons
}

\author{
Philippe de Forcrand ${ }^{\mathrm{a}} \mathrm{b} *$ and Michele Pepe ${ }^{\mathrm{a}}$ \\ anst. für Theoretische Physik, ETH Hönggerberg, CH-8093 Zürich, Switzerland \\ ${ }^{\mathrm{b}}$ CERN, Theory Division, CH-1211 Genève 23, Switzerland
}

We exhibit the connection between local gauge singularities in the Laplacian gauge and topological charge, which opens the possibility of studying instanton excitations without cooling. We describe our version of Laplacian gauge-fixing for $S U(N)$.

Topological excitations produce obstructions to making the gauge field smooth everywhere. Therefore, they should appear as singularities in an otherwise smooth gauge. This offers the possibility of identifying such excitations via gaugefixing. After gauge-fixing, the gauge field becomes singular, and the gauge ill-defined, on a sub-manifold characterizing the topological excitations. Even though the precise location of this manifold typically depends on the specific gauge condition chosen, its existence does not. This gauge-fixing approach was suggested by 't Hooft to identify chromo-magnetic monopoles [1]. It has been recognized recently that both monopoles and center vortices appear together as gauge singularities of co-dimension 3 and 2 respectively, when one tries to enforce a smooth gauge for the adjoint $S U(N) / Z_{N}$ field [2]. It is then natural to also study what happens when one tries to enforce a smooth gauge for the $S U(N)$ field. As we show below, point-like (co-dimension 4) singularities appear, coming from the topological charge of the Yang-Mills field. Thus, gaugefixing allows a unifying perspective on all 3 kinds of topological Yang-Mills excitations: center vortices, monopoles and instantons.

\section{Example: Landau gauge}

The gauge field of an $S U(2)$ instanton of size $\rho$ is

$A_{\mu}^{a}(x)=\eta_{\mu \nu}^{a} x_{\nu} \frac{2}{|x|^{2}+\rho^{2}}$ or $\bar{\eta}_{\mu \nu}^{a} x_{\nu} \frac{2 \rho^{2}}{|x|^{2}\left(|x|^{2}+\rho^{2}\right)}$

\footnotetext{
*Presented by Ph. de Forcrand at LAT00.
}

in regular and singular gauge respectively. Because of the antisymmetry of $\eta_{\mu \nu}$ and $\bar{\eta}_{\mu \nu}$, both expressions above satisfy the Landau gauge condition $\partial_{\mu} A_{\mu}=0$. Furthermore, starting from the singular gauge, one can apply the gauge transformation $\Omega(x)=\omega(x, b) \omega(x, 0)^{\dagger}$, where $\omega(x, y)=$ $\left((x-y)_{0} \mathbf{1}+i(x-y)_{i} \sigma_{i}\right) /|x-y|$, and still obtain a configuration which satisfies $\partial_{\mu} A_{\mu}=0$. In this configuration, the gauge field is singular at point $b$, which can be anywhere in 4-space. The regular gauge is just a special case where $b$ is sent to infinity. Therefore, Landau gauge is ambiguous on an instanton background field, and a whole family of Gribov copies exists. Nevertheless, the gauge field possesses a point-like singularity in each of these copies, whose location depends on the copy chosen. In the presence of small perturbations, the Gribov ambiguity would be resolved, but the location of the singularity would be dictated by the perturbation, and would have a priori little to do with the instanton center.

\section{Laplacian gauge: $S U(2)$}

On the lattice, the Landau gauge condition is usually translated into

$\sum_{x, \mu} \operatorname{Re} \operatorname{Tr} U_{x, \mu}$ maximum

which is ambiguous for any field configuration, due to the presence of local maxima called "lattice Gribov copies". In a gauge given by a local maximum, spurious singularities of the gauge field occur after continuum interpolation, which for instance bias the correlator $\left\langle A_{\mu}(0) A_{\mu}(x)\right\rangle$ [3]. Therefore, it is desirable to select a smooth gauge 
devoid of lattice Gribov copies to study gauge field singularities. The Laplacian gauge [4] is such a gauge. Like Landau gauge, it is smooth and Lorentz-symmetric, and fixes a pure gauge configuration $\left(F_{\mu \nu}=0\right)$ to $A_{\mu}=0$ (all links $\left.=\mathbf{1}\right)$. Our computer implementation for $S U(2)$ and $S U(3)$ uses the library ARPACK [5], and is much more economical than the iterative Landau gauge fixing. With it, a study of the infrared behaviour of the gluon propagator has been performed [6]. Unfortunately, the Laplacian gauge is intrinsically non-perturbative, which prevents a direct comparison with the Faddeev-Popov approach.

The Laplacian gauge condition consists of imposing, at every space-time point, a fixed color orientation for the eigenvector of the covariant Laplacian associated with the smallest eigenvalue. In other words, one determines the eigenvector $v(x)$ satisfying $\Delta(U) v(x)=\lambda_{0} v(x)$. At each point $x, v(x) \in C^{2}$ has 2 complex color components. The required gauge transformation $\Omega(x)$ is that which rotates $v(x)$ along some prescribed direction, say $(1,0)^{\dagger}$. The gauge thus specified is unique, except when the eigenvalue $\lambda_{0}$ is degenerate, which defines a genuine Gribov copy. 2

For a generic gauge field, the gauge may still be ill-defined locally: this happens at points $x$ where $\Omega(x)$ cannot be defined because $|v(x)|=0$. This implies 4 constraints: the sub-manifold where the gauge remains ambiguous consists of points in 4 dimensions. It is natural to examine the connection between such points and instantons.

Fig.1 shows $|v(x)|$ on an instanton configuration obtained by improved cooling [7]. $|v(x)|$ appears to vanish at a point which turns out to be very near the instanton center, and varies linearly with distance in its vicinity. This behaviour has been shown analytically, for the continuum theory in a finite volume, in [8]. Thus, one instanton can be identified via Laplacian gauge-fixing.

\section{Many instantons}

In the field of $Q$ instantons, one expects $Q$ gauge singularities in Laplacian gauge. Their locations cannot be obtained analytically, so we have performed numerical tests on configurations

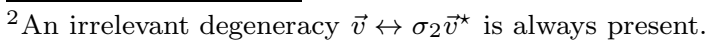

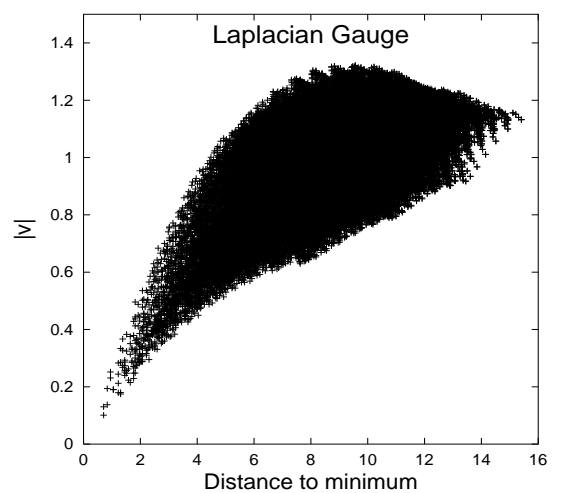

Figure 1. Magnitude of the lowest-lying eigenvector of the covariant Laplacian, for a cooled instanton gauge field.

of various topological content, obtained by improved cooling. To identify the location of the instantons, we use the "instanton finder" algorithm of [7], which looks for a local peak in the topological charge density, and checks self-duality near the peak. To identify the gauge singularity where the magnitude $|v(x)|$ of the Laplacian eigenvector vanishes, we fit $|v(x)|$ to the ansatz

$|v(x)| \sim v_{0}+\left|x-x_{0}\right| / r_{0}$

on a $3^{4}$ hypercube. If the fit is good $\left(\chi^{2} /\right.$ d.o.f. $\ll$ 1 , typically $\leq 0.004$ ) and $\left|v_{0}\right|$ is small (typically $\leq 0.25$ ), we assign a singularity at point $x_{0}$. Neighbouring $3^{4}$ cells usually detect the same singularity, providing a cluster of estimates $x_{0}$.

We find very good agreement between the locations of the topological excitations given by both methods. The agreement improves with cooling, and is worse on approximate instantons which depart more from a spherical ansatz. Even on a 7-instanton configuration, the agreement remains rather good, as shown in Fig.2.

When both instantons and anti-instantons are present, the agreement between the two methods deteriorates. The Laplacian method pays more attention to the global features of the gauge field, which all enter in the construction of the lowestlying eigenvector, and does not always "see" an instanton-antiinstanton pair. The constant $v_{0}$ in the ansatz eq.(3) helps towards accounting for the background of the opposite charge excitations. It is also possible to select an eigenvector 


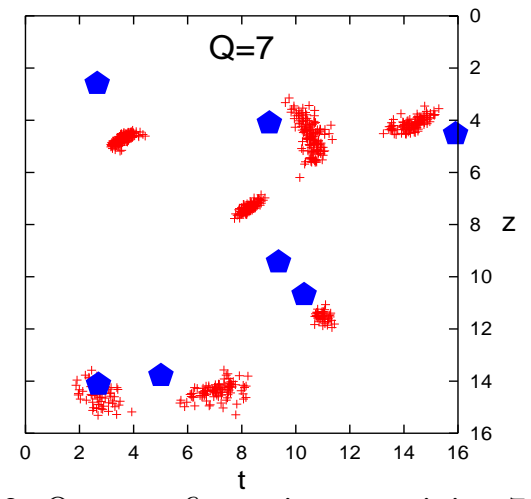

Figure 2. On a configuration containing 7 cooled instantons, comparison of their locations (pentagons) and the approximate zeros of the Laplacian eigenvector $(+)$, projected on the $(z, t)$ plane.

of the Laplacian associated with a larger eigenvalue, thus more sensitive to short-range gauge field fluctuations. The agreement with the usual method improves noticeably.

Similarly, one may try to analyze the topological content of a thermalized gauge field configuration. The usual method requires some cooling, which has been criticized because it destroys close instanton-antiinstanton pairs and distorts the remaining objects. In contrast, the Laplacian method filters the UV fluctuations automatically, so that information can be obtained without any cooling. This is illustrated in Fig.3. It is readily apparent that the two methods agree poorly in such circumstances.

This difference emphasizes once more the arbitrariness which necessarily enters any procedure which aims at identifying instantons and antiinstantons in a fluctuating ensemble. Strictly speaking, there is no such thing as an instantonantiinstanton pair, since it does not constitute a solution of the classical Yang-Mills equations. To distinguish such a pair from a topologically trivial fluctuation, one must separate non-perturbative and perturbative contributions by some criterion. This is a notoriously ambiguous proposition.

Therefore, one has here two methods, each containing some arbitrariness, to identify topological excitations. The usual method analyzes local features of the gauge field, and requires some arbitrary amount of cooling. The Laplacian method takes into account global features through the

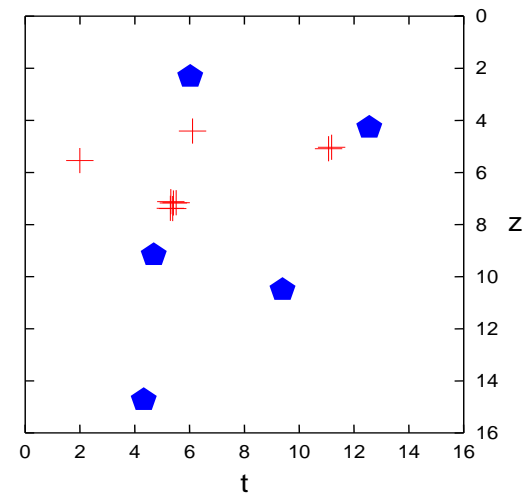

Figure 3. On a $\beta=2.4$ configuration, comparison of the locations of approximate instantons (pentagons) and of approximate zeros of the Laplacian eigenvector $(+)$, projected on the $(z, t)$ plane. The former required 30 cooling steps, the latter zero.

eigenvector. Which eigenvector one selects is arbitrary. In addition, both methods encapsulate further arbitrariness in the quality of the local fit required, in both cases, to identify an instanton.

One possible advantage we see to the Laplacian method is in the measurement of the total topological charge. Small instantons are easily destroyed in the early stage of cooling. The Laplacian method might give more reliable results. Of course the near-vanishing of $|v(x)|$ gives no indication on the sign of the associated topological charge, or the size and color orientation of the instanton. One must look at the gauge-fixed field to extract such information.

\section{Laplacian gauge: $S U(N)$}

A formulation of the Laplacian gauge for $S U(N)$ was given in 4 . It requires the determination of the $N$ lowest-lying eigenvectors of the covariant Laplacian. At each point $x$, the $N \times N$ complex matrix $M$ whose columns are formed by these eigenvectors is projected onto $S U(N)$, using the polar decomposition $M=W P, W \in U(N), P$ $=\left(M^{\dagger} M\right)^{1 / 2}$. The required gauge transformation is then $\Omega(x)=e^{i \alpha} W^{\dagger}$, where $\alpha=\frac{1}{N} \arg (\operatorname{det} W)$. $\Omega(x)$ rotates $M$ "parallel" to the identity $\mathbf{1}_{N}$.

Here we use a different $S U(N)$ formulation, which requires $(N-1)$ eigenvectors only. Moreover, in this formulation, the connection between 
topological charge and local gauge ambiguities remains transparent. We consider the $(N-1)$ eigenvectors $v_{1}, v_{2}, . ., v_{N-1}$ in succession, and associate a partial gauge-fixing with each.

i) At each point $x$, rotate $v_{1}(x)$ parallel to $(1,0, . ., 0)_{N}^{\dagger}$. The corresponding gauge transformation $\Omega_{1}(x)$ is not uniquely defined. Any $S U(N-1)$ transformation among the components $2,3, . ., N$ can still be performed. This is consistent with the number of remaining degrees of freedom: $(2 N-1)$ constraints must be satisfied to bring $v_{1}(x)$ parallel to a reference direction, which leaves $\left(N^{2}-1\right)-(2 N-1)=(N-1)^{2}-1$ d.o.f., corresponding to an $S U(N-1)$ gauge freedom. Choose any satisfactory $\Omega_{1}$ and rotate all eigenvectors $v_{1}, v_{2}, . ., v_{N-1}$.

ii) Rotate the last $(N-1)$ components of $v_{2}(x)$ to make it parallel to $(1,0, . ., 0)_{N-1}^{\dagger}$, leaving the first component untouched. This defines a gauge transformation $\Omega_{2}(x) \in \mathbf{1} \times S U(N-1)$, up to a further $S U(N-2)$ transformation. Choose any satisfactory $\Omega_{2}$ and rotate all eigenvectors.

iii) Rotate the last $(N-2)$ components of $v_{3}(x)$ parallel to $(1,0, . ., 0)_{N-2}^{\dagger}$, etc...

$i v$ ) Finally the last 2 components of $v_{N-1}(x)$ are rotated parallel to $(1,0)^{\dagger}$, defining a gauge transformation $\Omega_{N-1}(x) \in \mathbf{1}_{N-2} \times S U(2)$, as in Section 2 , and completing the gauge fixing.

The complete gauge transformation is therefore $\Omega_{N-1} \times \ldots \times \Omega_{2} \times \Omega_{1}$. It turns the matrix $M$ formed by $v_{1}, v_{2}, . ., v_{N}$ to upper triangular form, regardless of the value of $v_{N}$, which needs not be computed. Our procedure can be viewed as a $Q R$ decomposition of $M$.

Let us now consider the ambiguities associated with our $S U(N)$ procedure.

- Each eigenvector is defined up to a complex phase, which is global and will induce an irrelevant, global Abelian gauge transformation.

- The gauge becomes ambiguous when any of the lowest $(N-1)$ eigenvalues is degenerate, preventing a unique determination (up to a phase) of the eigenvectors. This defines a genuine Gribov copy. In this case, a continuous family of gauges is obtained, corresponding to different choices of orthogonal eigenvectors in the degenerate subspace. - On a generic field configuration, local gauge ambiguities arise at points $x$ where all the last $(N-k+1)$ complex components of $v_{k}(x)$ vanish, making the gauge transformation $\Omega_{k}(x)$ illdefined. This implies $2(N-k+1)$ real constraints, thus defining defects of co-dimension $2(N-k+1)$.

These defects all have a topological meaning. For example, $S U(5)$ will admit point-like defects in $d=10$ dimensions $(N=5, k=1)$, consistent with the non-trivial homotopy group $\Pi_{2 N-1}(S U(N))=Z$. Even though such excitations are irrelevant classically because, in $d>4$, their action decreases with their size, their role could be studied non-perturbatively via Laplacian gauge-fixing.

In 4 dimensions, the only defects of codimension $\leq 4$ are point-like, and come from the last step in our gauge-fixing procedure, which involves an $S U(2)$ gauge transformation. Thus, one recovers the expected result that the topological charge of an $S U(N)$ configuration can be "squeezed" to an $S U(2)$ subgroup [even if the instantons themselves may not be], where it gives rise to local singularities in a smooth gauge. Again, this offers the possibility of extracting the topological charge of an $S U(N)$ configuration without cooling.

Acknowledgments: We thank C. Alexandrou, J.L.F. Barbón, F. Bruckmann, J. Fröhlich, M. García Pérez, M. Lüscher and A. Wipf for correspondence and discussions.

\section{REFERENCES}

1. G. t'Hooft, Nucl. Phys. B190 (1981) 455.

2. Ph. de Forcrand and M. Pepe, heplat/0008014, hep-lat/0008016.

3. Ph. de Forcrand and J. E. Hetrick, Nucl. Phys. B (Proc. Suppl.) 42 (1995) 861.

4. J.C. Vink and U.-J. Wiese, Phys. Lett. B289 (1992) 122.

5. http://www.caam.rice.edu/software/ARPACK/.

6. C. Alexandrou, Ph. de Forcrand and E. Follana, hep-lat/0008012; hep-lat/0009003.

7. Ph. de Forcrand et al., Nucl. Phys. B 499 (1997) 409.

8. F. Bruckmann et al., hep-th/0007119. 